\title{
Deep Learning based Segmentation for Multi MR Imaging Protocols using Transfer Learning for PET Attenuation Correction
}

\author{
$1^{\text {st }}$ Imene Mecheter \\ Department of Electronics and Computer Engineering \\ Brunel University London \\ Uxbridge, UK \\ imene.mecheter@brunel.ac.uk \\ $3^{\text {rd }}$ Maysam Abbod \\ Department of Electronics and Computer Engineering \\ Brunel University London \\ Uxbridge, UK
}

\author{
$2^{\text {nd }}$ Abbes Amira \\ Institute of Artificial Intelligence \\ De Montfort University \\ Leicester, UK
}

\author{
$4^{\text {th }}$ Habib Zaidi \\ Division of Nuclear Medicine and Molecular Imaging \\ Geneva University Hospital \\ Geneva, Switzerland
}

\begin{abstract}
Magnetic resonance (MR) image segmentation is a robust technique used for PET attenuation correction. However, the segmentation of the brain into different tissue classes is a challenging task because of the similarity of pixel intensity values. The objective of this work is to propose a deep learning network to segment T1-weighted MR images of a dataset consists of 50 patients. Additionally, transfer learning is applied to segment another MR image protocol which is T2-weighted. The pretrained network with T1-weighted images is finetuned then tested with a dataset of 14 patients only. The Dice coefficients of air, soft tissue, and bone classes for T1-weighted MR images are $0.98,0.92$, and 0.79 respectively. The results of transfer learning show the feasibility of finetuning a deep network trained with T1-weighted images to segment T2-weighted images.

Index Terms-Magnetic Resonance Imaging, Segmentation,
\end{abstract} PET Attenuation Correction, Deep Learning, Transfer Learning

\section{INTRODUCTION}

Positron emission tomography (PET) and magnetic resonance (MR) are two complemantry imaging modalities that provide physiological and morphological information of different tissues in both normal and pathological cases. Recently, the hybrid PET/MR imaging system has been commercialized and adopted in clinical domain [1]. The main challenge is the PET quantification process which requires the photon attenuating properties which cannot be derived directly from MR image intensitties. The attenuation maps are commonly derived from computed tomography (CT) images because of the direct mapping between $\mathrm{CT}$ intensities and attenuation coefficients. Therefore, it is necessary to explore more complicated ways to convert the MR image intensities to $511 \mathrm{KeV}$ attenuation maps [2].

Three methods have been performed to address the PET attenuation correction problem using MR imaging which are: segmentation, atlas, and emission methods [2]. The segmen- tation based method is considered the most robust and simple method which has been deployed in commercial scanners and introduced in clinical practice [2]. Atlas based method shows its superiority, however, it is not considered yet as a robust method to be introduced in clinical domain [3].

Deep learning networks have been recently applied on various computer vision applications. In the medical domain, different deep network architectures have been proposed for medical images segmentation [4] such as Fully convolutional network [5], Segnet [6], and U-Net [7].

Deep networks are performing greatly with the assumption that the training and testing datasets follow the same data distribution. However, this is very challenging with medical datasets due to the variation of commercial scanners and the availability of different imaging modalities and protocols. This variation is called domain shift [8]. Figure 1 shows the visual comparison between T1-weighted (T1-w) MR protocol, T2weighted (T2-w) MR protocol, and CT image where T1-w MR image looks darker than T2-w in most tissue classes.

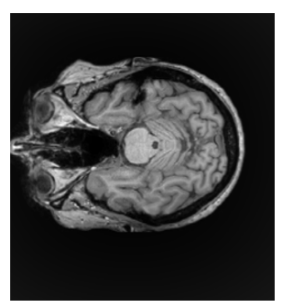

T1 weighted MR

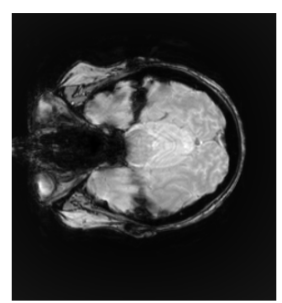

T2 weighted MR

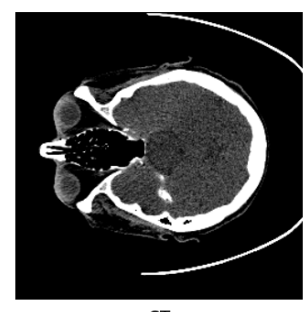

CT
Fig. 1. Example of training datasets: T1-w and T2-w MR images with their corresponding CT image.

In this work, a deep convolutional auto encoder network is applied to perform brain MR image segmentation into three 
tissue classes. The network is trained from scratch using T1-w MR images. Afterwards, transfer learning is applied to finetune the pretrained network to segment T2-w MR images.

The structure of the paper is as follows: section 2 describes the data. Section 3 presents the proposed methodology. Section 4 illustrates the experimental results and evaluation. Finally the conclusion is covered in section 5 .

\section{DATA}

\section{A. Data acquisition}

Brain MR and PET/CT images are acquired as part of the clinical workup of patients. The dataset consists of 50 patients of T1-w MR images and 14 patients of T2-w MR images. The patients images show clinical indication of dementia $(70 \%)$, epilepsy $(25 \%)$ and brain tumors $(5 \%)$. The age range of the patients is $64.6 \pm 11.7$ years.

At the first step, the patients underwent an MRI scan on a 3T Siemens MAGNETOM Skyra scanner with a 64 channel head coil. The MR images scans used for this study are 3D T1- w magnetization prepared rapid gradient-echo, MP-RAGE (TE/TR/TI, $2.3 \mathrm{~ms} / 1900 \mathrm{~ms} / 970 \mathrm{~ms}$, flip angle 8 ; NEX = 1 , voxel size $\left.0.8 \times 0.8 \times 0.8 \mathrm{~mm}^{3}\right)$ and $3 \mathrm{D} \mathrm{T} 2-\mathrm{w}$ turbo spinecho, TSE (TE/TR, $100 \mathrm{~ms} / 6200 \mathrm{~ms}$, NEX = 2; voxel size $\left.0.4 \times 0.4 \times 4 \mathrm{~mm}^{3}\right)$. Afterwards, the patients underwent an $18 \mathrm{~F}$ FDG PET/CT scan on the Siemens Biograph mCT scanner for 20 min after injection of $210.2 \pm 13.9 \mathrm{MBq}$ 18F-FDG.

\section{B. Data preprocessing}

The MR volumes of both T1-w and T2-w are converted into 2D slices. The number of slices per each volume is different and the dimension of each slice is $512 \times 512$. Each slice is cropped into a $256 \times 256$ image to remove the background. Then, local contrast normalization technique is applied on each image. The same processing steps are applied to CT volumes which are used as ground truth. The total number of 2D images for the whole dataset is 2982 slices. Among them, only 2328 slices are used after discarding some of the first and last slices of each volume. The discarded slices have just few pixels that correspond to the brain tissue while the majority of the pixels correspond to the background which is not useful for the training process.

\section{Ground truth generation}

CT images are used as ground truth for the supervised training process. MR images are firstly co-registered with CT images to determine a common coordinate system that enables the pixel-based comparison of images. Each MR slice is coregistered with its corresponding CT slice by applying the rigid Euler transformation followed by the non-rigid B-spline transformation using Elastix tool [9]. Afterward, simple pixel intensity-based thresholding is applied to create the CT ground truth. The CT image is segmented into three tissue classes which are air, bone, and soft tissue. Hounsfield values which are: greater than $600 \mathrm{HU}$ are classified as bone, lower than $-500 \mathrm{HU}$ are classified as air, otherwise are labeled as soft tissue.

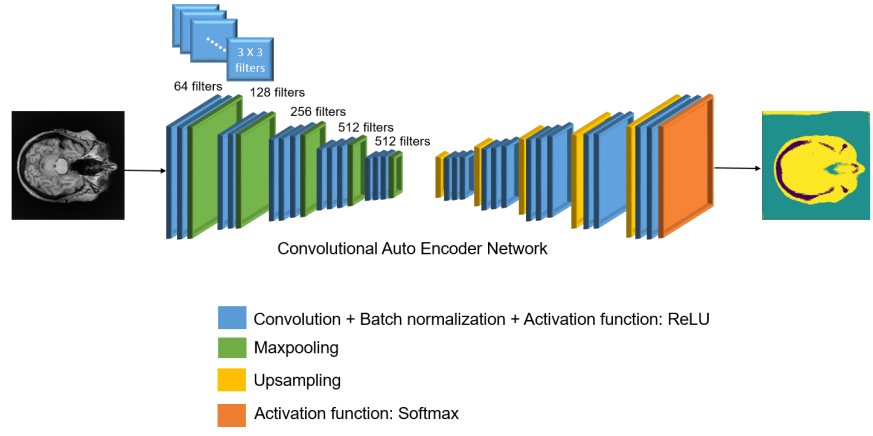

Fig. 2. An illustration of the SegNet architecture

\section{PRoposed Methodology}

The proposed method consists of two parts. The first part is training a deep convolutional auto encoder (CAE) network from scratch to segment T1-w MR images into three tissue classes. The second part is finetuning the pre-trained network with T1-w images to segment T2-w MR images.

\section{A. Deep CAE Network to segment T1-w MR}

The segmentation network architecture is based on the Segnet which follows the convolutional auto encoder architecture. SegNet is one common deep CAE model that has been applied successfully to segment the brain MR images [10], [11]. This model consists of an extracting path to capture the features and an expanding path for localization and resizing. The output of such networks preserves the same size of the input after extracting the meaningful features. The extracting path consists of 13 convolutional layers with its corresponding batch normalization layers, maxpooling layers, and rectifiedlinear unit (ReLU) activation function. Each convolutional layer consists of multiple $3 \times 3$ filters. The expanding path is the mirror of the extracting path with upsampling layers to retrain the original size of the input. The final layer of the network is a multiclass softmax classifier that produces class probabilities for each pixel. Figure 2 illustrates the network architecture.

The network is trained from scratch using T1-w MR datasets using two folds cross validation. A grid search strategy is followed to find the best training parameters. The network weights are initialized using $\mathrm{He}$ normal scheme [12] and updated using Adam optimizer with a learning rate that starts with 0.001 then reduces when there is no improvement on the training loss for more than 10 epochs. Beta 1 and Beta 2 which are the parameters of Adam's optimizer that controls the decay rates are set to 0.9 and 0.999 respectively. The network reads 10 samples per each batch and calculates the multiclass crossentropy loss for 300 epochs. Dropout with a factor of 0.7 is applied to reduce the overfitting.

\section{B. Transfer learning to segment T2-w MR}

The pretrained trained model with T1-w MR images is used as the base model to train a small dataset of $\mathrm{T} 2-\mathrm{w}$ MR images. The base model is finetuned by freezing the 


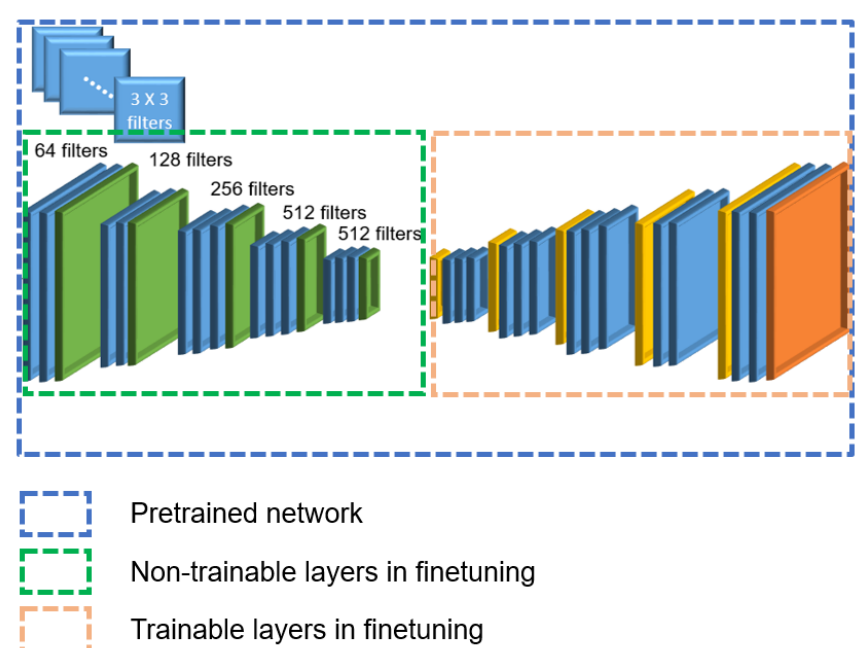

Fig. 3. An illustration of the transfer learning process to segment T2-w MR images

extracting path while re-training the expanding path. The process of freezing the parameters of specific layers preserves the extracted features from T1-w MR images. The learning rate is set to a smaller value (1e-4) than the learning rate of the pretrained model to avoid destroying the learnt features. The number of epochs required to finetune the model is only 30 epochs. Figure 3 illustrates the transfer learning process.

\section{Computing Environment}

MATLAB and Python programming languages are used to build the proposed method. The data preprocessing is performed using MATLAB while Python libraries: Keras and TensorFlow are used for deep learning. The deep network model is trained using Tesla V100 GPU with 16 GB RAM as part of "Raad2" GPU cluster.

\section{EXPERIMENTAL RESUlts AND EVAluation}

The first conducted experiment is the training and testing of the Segnet network to segment T1-w MR images into three different tissue classes: air, soft tissue, and bone. Then, the second experiment is the application of transfer learning by finetuning the pretrained model with T1-w images to fit the T2-w images.

\section{A. Results of the segmentation of T1-w MR images}

The segmentation results of T1-w MR images are illustrated in Figure 4. The first row corresponds to three input slices, the second row shows their corresponding CT images as ground truth, and the last row represents the results of the segmentation. Table I shows the segmentation evaluation metrics of testing dataset from T1-w MR testing images.

The air and soft tissue classes achieve higher segmentation dice than the bone class. The challenging segmentation of bone tissue originates from the imbalance tissue classes as such the total number of air and soft tissue pixels per each slice is much higher than the bone pixels.
A

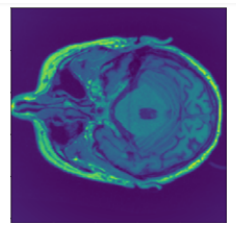

B

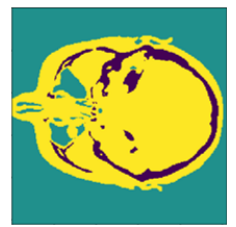

C

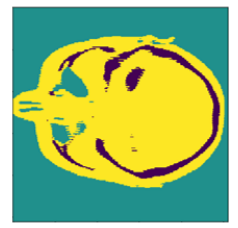

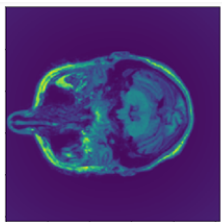
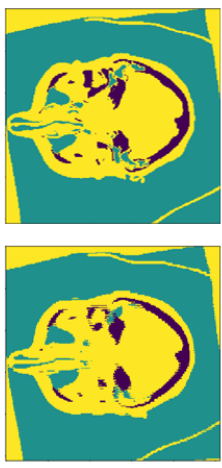
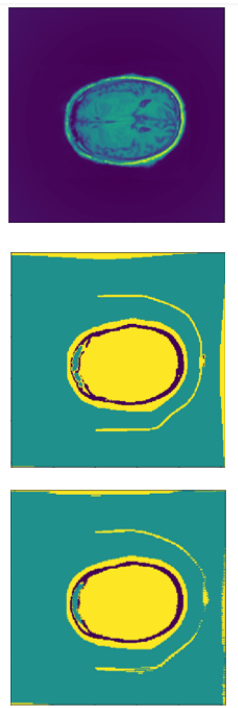

Fig. 4. Example of three MR T1-w slices (A) with their corresponding CT slices as ground truth (B) and the automatic segmented slices using deep learning $(\mathrm{C})$. The colors in row $\mathrm{B}$ and $\mathrm{C}$ refer to the following classes: green is the air class, yellow represents the soft tissue class, and the purple shows the bone class.

TABLE I

EVALUATION METRICS OF THE SEGMENTATION RESULTS OF BRAIN T1-W MR IMAGES

\begin{tabular}{|c||c||c||c|}
\hline Metric & Air & Soft tissue & Bone \\
\hline Precision & 0.98 & 0.93 & 0.80 \\
\hline Recall & 0.98 & 0.93 & 0.77 \\
\hline Dice & 0.98 & 0.92 & 0.79 \\
\hline Jaccard Index & 0.96 & 0.86 & 0.65 \\
\hline
\end{tabular}

\section{B. Results validation}

The obtained segmentation results are validated with two other approaches that applied deep learning to segment the brain MR images for PET attenuation correction. Following the network architecture and training options adapted from [10], a segnet network is trained using our datasets. The second approach [13] applies the same segnet network architecture with the addition of conditional random field as a post processing setp. The segmentation results of each approach are illustrated in table II.

\section{Results of transfer learning and finetuning}

The results of the segmentation of T2-w MR images are shown in Figure 5. Table III illustrates the dice similarity

TABLE II

COMPARISON WITH OTHER DEEP LEARNING BASED SEGMENTATION METHODS

\begin{tabular}{|c||c||c||c|}
\hline Method & Air & Soft tissue & Bone \\
\hline Segnet [10] & 0.97 & 0.90 & 0.66 \\
\hline Segnet+ CRF [13] & 0.84 & 0.57 & 0.07 \\
\hline Proposed method & $\mathbf{0 . 9 8}$ & $\mathbf{0 . 9 2}$ & $\mathbf{0 . 7 9}$ \\
\hline
\end{tabular}


A

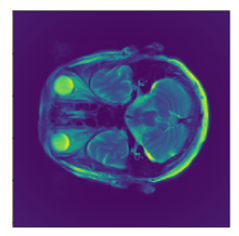

B

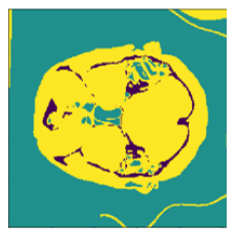

C

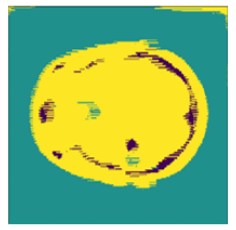

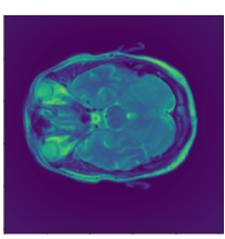
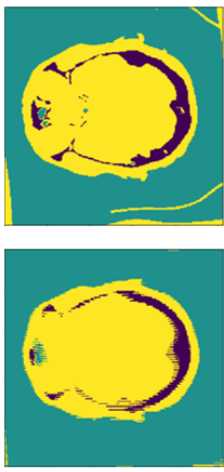
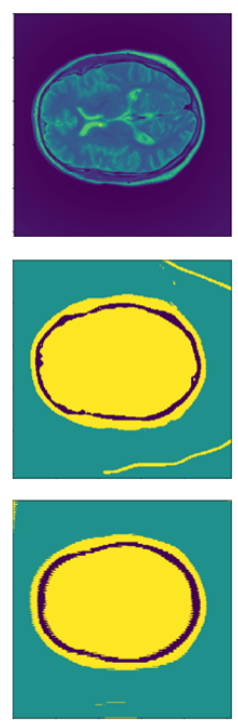

Fig. 5. The segmentation results of T2-w MR images using transfer learning. Three MR T2-w slices are shown in row (A) with their corresponding CT slices as ground truth (B) and the automatic segmented slices using transfer learning $(\mathrm{C})$. The colors in row $\mathrm{B}$ and $\mathrm{C}$ refer to the following classes: green is the air class, yellow represents the soft tissue class, and the purple shows the bone class.

TABLE III

THE DICE SIMILARITY COEFFICIENT OF THE T2-W MR BRAIN TISSUE CLASSES: AIR, SOFT TISSUE, AND BONE USING TRANSFER LEARNING AND FINETUNING THE T1-W PRETRAINED MODEL.

\begin{tabular}{|c||c||c||c|}
\hline & Air & Soft tissue & Bone \\
\hline Transfer learning & 0.97 & 0.85 & 0.51 \\
\hline Finetune: 2 CONV & 0.97 & 0.86 & 0.50 \\
\hline Finetune: 4 CONV & 0.97 & 0.87 & 0.58 \\
\hline Finetune: 6 CONV & 0.97 & 0.87 & 0.60 \\
\hline Finetune: 8 CONV & 0.97 & 0.87 & 0.60 \\
\hline Finetune: Decoder & $\mathbf{0 . 9 7}$ & $\mathbf{0 . 8 7}$ & $\mathbf{0 . 6 3}$ \\
\hline
\end{tabular}

coefficient of the three classes. The first row in the table refers to the results of transfer learning where the pretrained model is used as it is without any finetuning. The results show that there is an improvement in the segmentation results as we finetune more convolutional layers of the pretrained model. The best results are achieved by freezing the layers of the extracting path (encoder) and retraining the layers of the expanding path (decoder).

Like T1-w MR image segmentation, the air class achieves the highest dice value followed by the the soft tissue class then the bone class. Despite the finetuning of more convolutional layers helps to improve the segmentation accuracy of both the soft tissue and bone class, the air class segmentation does not show any improvement.

The training time varies from training a network from scratch to finetuning a pretrained model. The approximate required time of training T1-w MR images from scratch is 6 hours. However, the finetuning of the pretrained model requires only 10 minutes. Moreover, the predication time is

very short such as labeling a single slice takes only $6 \mathrm{~ms}$.

\section{CONCLUSION}

In this study, an MR based attenuation correction method using deep learning has been applied to segment the brain MR images into three tissue classes: air, soft tissue and bone. Transfer learning technique is applied to show the feasibility of finetuning a model trained with T1-w MR images to segment T2-w MR images. The promising results indicate that there is a possibility to build a model that can be applied to segment MR images with different MR protocols. The successful application of deep learning can improve the segmentation accuracy and hence improves the PET attenuation correction and quantification.

\section{ACKNOWLEDGMENT}

The authors extend a special thanks to Texas A\&M at Qatar's Advanced Scientific Computing (TASC) center who provided the access and technical support to the High Performance Computing cluster "Raad2".

\section{REFERENCES}

[1] Z. Chen, S. D. Jamadar, S. Li, F. Sforazzini, J. Baran, N. Ferris, N. J. Shah, and G. F. Egan, "From simultaneous to synergistic mr-pet brain imaging: A review of hybrid mr-pet imaging methodologies," Human brain mapping, vol. 39, no. 12, pp. 5126-5144, 2018.

[2] A. Mehranian, H. Arabi, and H. Zaidi, "Vision 20/20: Magnetic resonance imaging-guided attenuation correction in PET/MRI: Challenges, solutions, and opportunities," Medical Physics, vol. 43, no. 3, pp. 11301155, Mar. 2016.

[3] K. Shi, S. Fürst, L. Sun, M. Lukas, N. Navab, S. Förster, and S. I. Ziegler, "Individual refinement of attenuation correction maps for hybrid pet $/ \mathrm{mr}$ based on multi-resolution regional learning," Computerized Medical Imaging and Graphics, vol. 60, pp. 50-57, 2017.

[4] Z. Akkus, A. Galimzianova, A. Hoogi, D. L. Rubin, and B. J. Erickson, "Deep learning for brain mri segmentation: state of the art and future directions," Journal of digital imaging, vol. 30, no. 4, pp. 449-459, 2017.

[5] J. Long, E. Shelhamer, and T. Darrell, "Fully convolutional networks for semantic segmentation," in Proceedings of the IEEE conference on computer vision and pattern recognition, 2015, pp. 3431-3440.

[6] V. Badrinarayanan, A. Kendall, and R. Cipolla, "Segnet: A deep convolutional encoder-decoder architecture for image segmentation," IEEE transactions on pattern analysis and machine intelligence, vol. 39, no. 12, pp. 2481-2495, 2017.

[7] O. Ronneberger, P. Fischer, and T. Brox, "U-net: Convolutional networks for biomedical image segmentation," in International Conference on Medical image computing and computer-assisted intervention. Springer, 2015, pp. 234-241.

[8] T. Tommasi, M. Lanzi, P. Russo, and B. Caputo, "Learning the roots of visual domain shift," in European Conference on Computer Vision. Springer, 2016, pp. 475-482.

[9] S. Klein, M. Staring, K. Murphy, M. A. Viergever, and J. P. Pluim, "Elastix: a toolbox for intensity-based medical image registration," IEEE transactions on medical imaging, vol. 29, no. 1, pp. 196-205, 2010.

[10] F. Liu, H. Jang, R. Kijowski, T. Bradshaw, and A. B. McMillan, "Deep learning $\mathrm{mr}$ imaging-based attenuation correction for pet/mr imaging," Radiology, vol. 286, no. 2, pp. 676-684, 2017.

[11] H. Jang, F. Liu, G. Zhao, T. Bradshaw, and A. B. McMillan, "Technical Note: Deep learning based MRAC using rapid ultrashort echo time imaging," Medical Physics, vol. 45, no. 8, pp. 3697-3704, 2018.

[12] K. He, X. Zhang, S. Ren, and J. Sun, "Delving deep into rectifiers: Surpassing human-level performance on imagenet classification," in Proceedings of the IEEE international conference on computer vision, 2015, pp. 1026-1034.

[13] H. Jang, F. Liu, G. Zhao, T. Bradshaw, and A. B. McMillan, "Deep learning based mrac using rapid ultrashort echo time imaging," Medical physics, vol. 45, no. 8, pp. 3697-3704, 2018. 